\title{
Complications associated with traction on the hip during arthroscopy
}
G. A. Elsaidi,
D. S. Ruch,
W. D. Schaefer,
K. Kuzma,
B. P. Smith

From Wake Forest

University School of

Medicine, Winston-

Salem, USA
G. A. Elsaidi, DO, Resident Orthopaedic Surgery

D. S. Ruch, MD, Associate Professor

K. Kuzma, BS, Medical

Student

B. P. Smith, PhD, Associate

Professor

Department of Orthopaedics,

Wake Forest University

School of Medicine, Medical

Centre Boulevard, Winston-

Salem, North Carolina 27157, USA.

W. D. Schaefer, MD, Orthopaedic Surgeon Fayetteville Orthopedic Clinic, 3308 Melrose Street, Fayetteville, North Carolina, USA.

Correspondence should be sent to $\operatorname{Dr}$ G. A. Elsaidi.

C2004 British Editorial Society of Bone and Joint Surgery doi:10.1302/0301-620X.86B6. $14426 \$ 2.00$

$J$ Bone Joint Surg [Br] 2004;86-B:793-6.

Received 15 April 2003

Accepted after revision

6 October 2003

We studied 16 hips (eight cadaver specimens) using arthrography, arthroscopy and anatomical dissection, under incremental traction of up to a maximum of $64 \mathrm{~kg}$, to determine the relationship of the portals to nearby neurovascular structures.

The distance of each arthroscopic portal (anterior, anterolateral, and posterolateral) to the associated neurovascular structures was measured after the application of $23 \mathbf{~ k g}$ of traction.

Traction of up to $\mathbf{6 4} \mathrm{kg}$ on the lower limb failed to produce evidence of labral or capsular injury. Furthermore, traction of $23 \mathrm{~kg}$ resulted in little change in the position of adjacent neurovascular structures relative to the standard arthroscopic portals.

Reports of the use of arthroscopy in the diagnosis and management of inflammatory, degenerative, and traumatic conditions of the hip have increased in the last decade. ${ }^{1-8}$ The complications associated with the procedure include injury to the sciatic, lateral femoral cutaneous and pudendal nerves, and potential injury to the acetabular labrum. ${ }^{9-11} \mathrm{~A}$ longitudinal force of at least $20 \mathrm{~kg}$ may be required to obtain a good view of the joint. ${ }^{10,12}$ Dorfmann and Boyer ${ }^{13}$ noted that this amount of traction may fix and lateralise the neurovascular structures thus making them more susceptible to injury. However, the effects of traction on the integrity of the joint capsule, the acetabular labrum and the ligamentum teres, remain unknown.

We studied the neurovascular structures relative to the three standard arthroscopic portals in order to determine the effect of traction on their spatial relationship, and to examine the effect of increasing traction on the capsule of the hip, the acetabular labrum and the ligamentum teres.

\section{Methods and Materials}

We used ten fresh frozen cadavers, with a mean body-weight of $72 \mathrm{~kg}$ (54 to 99). All were removed from refrigeration 13 hours before use, and the experiments were carried out at room temperature.

The cadavers were placed in the supine position on a radiolucent fracture table with a padded perineal post. Steinmann pins of 4.4 $\mathrm{mm}$ in width were placed through each distal femur, and traction bows were fixed to the pins (Fig. 1). A spring scale was placed directly in line with the traction bow and a force of $12 \mathrm{~kg}$ was applied. Iodinated contrast arthrography was performed through a medial approach to the hip in order to examine the integrity of the capsule and to confirm the absence of a radiographically apparent lesion of the labrum. Frank labral tears were seen in two specimens and these were therefore excluded from further tests. In the remaining eight cadavers (16 hips), a distracting force was applied to the lower limb in increments of $2 \mathrm{~kg}$ to a maximum of 64 $\mathrm{kg}$. After each increment, radiography was carried out to ascertain if there was damage to the capsule or labrum. After the application of traction of $64 \mathrm{~kg}$ this was reduced to $23 \mathrm{~kg}$ and arthroscopy was carried out using the anterior, anterolateral and posterolateral portals as described by Byrd, Pappas and Pedley. ${ }^{14}$ The capsule, the labrum and the ligamentum teres were examined for evidence of damage.

The anterolateral portal was placed at the anterolateral corner of the greater trochanter and directed parallel to the femoral neck at the lateral margin of the capsule (Fig. 1). The anterior portal was made at the intersection of a sagittal line drawn distally from the anterosuperior iliac spine and a perpendicular line drawn across the superior margin of the greater trochanter. The portal was then directed $45^{\circ}$ cephalad and towards the midline. The posterolateral portal was placed at the posterolateral corner of the greater trochanter and passed parallel to the femoral neck into the 


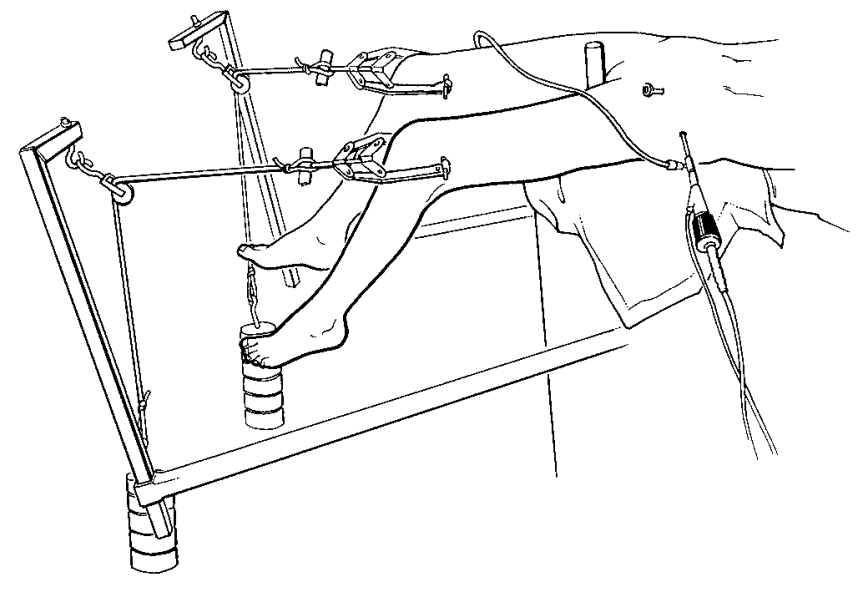

Fig. 1

Diagram showing the traction apparatus with an arthroscope in the anterolateral portal and a cannula in the anterior portal.

posterior margin of the lateral capsule. The skin was incised with a No. 11 blade. A spinal needle was inserted and a wire was passed through it. The spinal needle was then retracted and removed over the wire. A blunt $5.0 \mathrm{~mm}$ cannulated trocar/sheath assembly (Dyonics, Andover, Massachusetts) was passed over the wire (Fig. 1) which was then removed. The location of the needle for the anterior and posterolateral portals was confirmed through the arthroscope introduced from the anterolateral portal, before placement of the blunt trocars. Arthroscopic cameras (30E and 70E; Dyonics) were used to verify the integrity of the capsule, the labrum and the ligamentum teres.

After the arthroscopic examination, Steinmann pins were placed through the arthroscopic sheaths to maintain their intra-articular positions. The hip was then dissected and the lateral femoral cutaneous nerve, the lateral femoral circumflex artery, the sciatic nerve, the superior gluteal artery and nerve, and the femoral artery and nerve were identified. The position of the arthroscopic portals in relation to these neurovascular structures was measured, under 3.5 loupe magnification to the nearest $0.1 \mathrm{~mm}$, using calipers. Measurements were made of the distance between any branch of the lateral femoral cutaneous nerve and the anterolateral portal. Then, the distraction of $23 \mathrm{~kg}$ was released, and the measurements were repeated without traction.

Finally, the capsule of the hip was defined and its integrity confirmed visually. The joint was opened and the labrum was inspected. Small pieces $(1.0 \mathrm{~cm}$ x $1.5 \mathrm{~cm})$ were taken from the labrum and the anterior, anterolateral, and posterior aspects of the capsule for histological study. The sections were stained with haematoxylin and eosin and examined for the presence or absence of microscopic tears. Statistical analysis. This was performed using paired Student's $t$-test and a two-way analysis of variance (ANOVA).

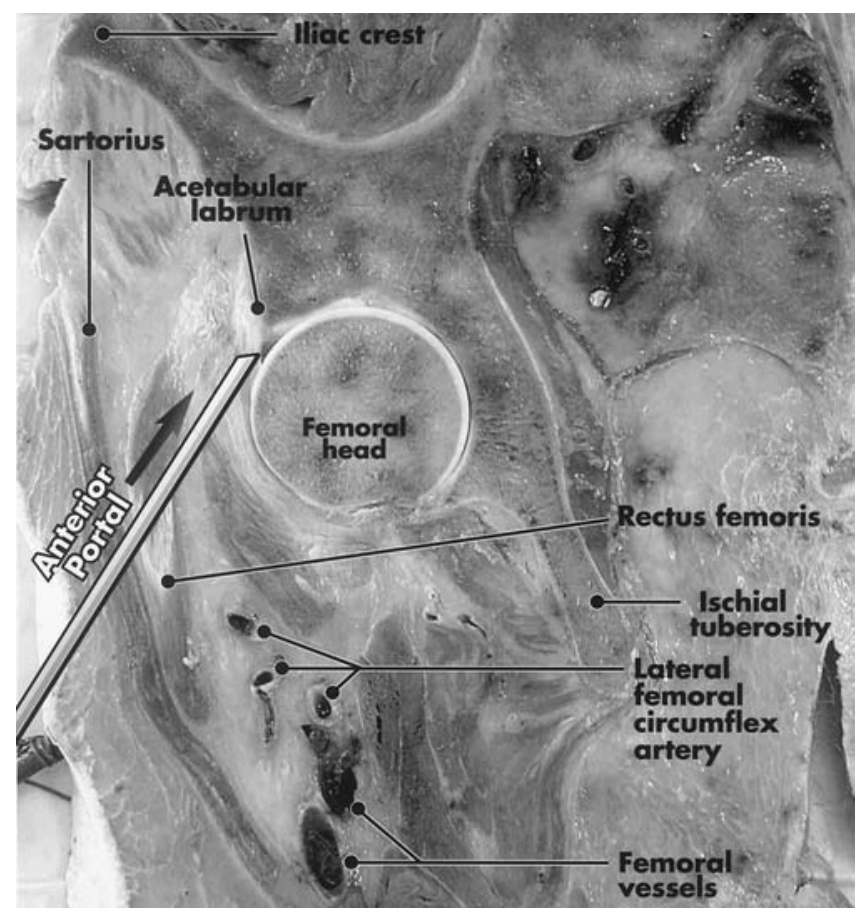

Fig. 2

Coronal section through the anterior cannula as it passes through sartorius, rectus femoris, and away from the lateral femoral circumflex vessels.

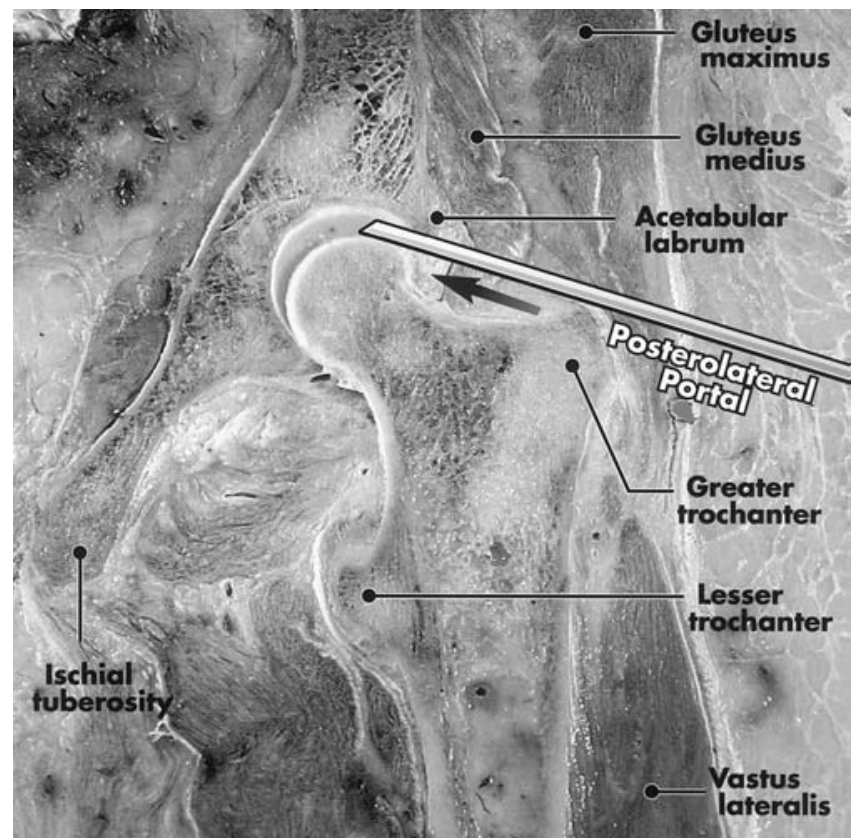

Fig. 3

Coronal section through the posterolateral portal as it passes beneath the edge of the acetabular labrum. This portal passes through gluteus maximus and gluteus medius. 


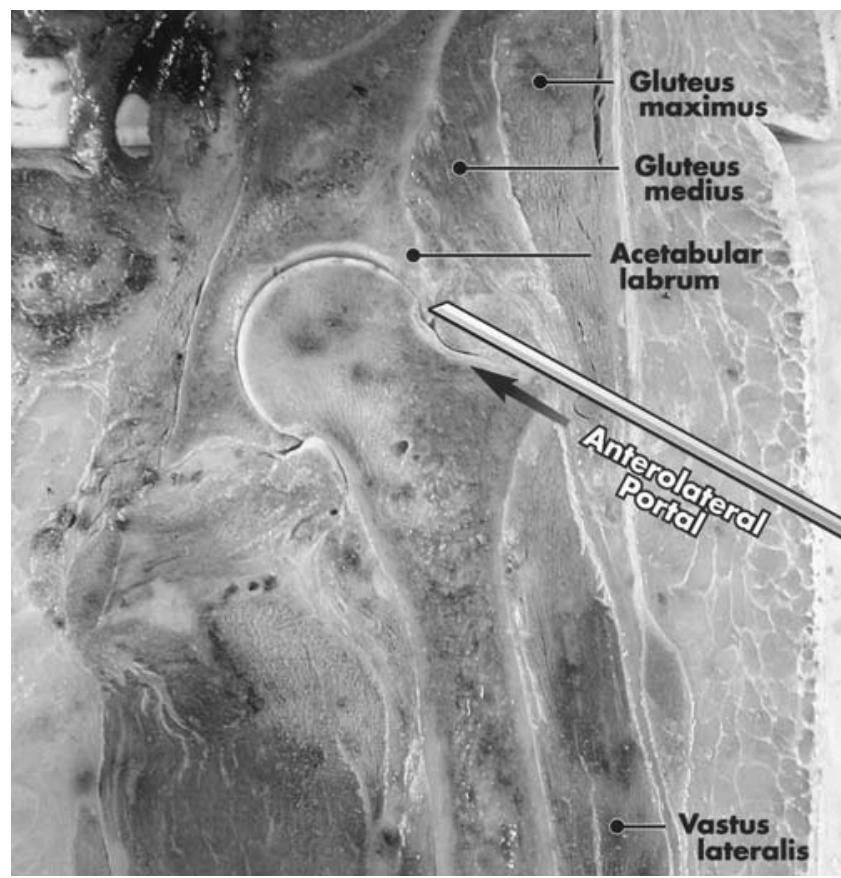

Fig. 4

Coronal section taken after placement of a cannula in the anterolateral portal. The plane of the section passes trough the tensor fascia lata and gluteus medius as it passes under the acetabular labrum.

\section{Results}

Relationship of portals to the neurovascular structures without traction

Anterior portals (Fig. 2). The trocar passed through the lateral aspect of sartorius and rectus femoris. The portal was located at a mean distance of $5.0 \mathrm{~mm}$ (0 to 28) from the lateral femoral cutaneous nerve, $24 \mathrm{~mm}$ (5 to 48) from the femoral nerve and $39 \mathrm{~mm}$ (15 to 80 ) from the femoral artery.

Posterolateral portal. The trocar passed posterior to the distal margin of the greater trochanter through gluteus medius and minimus, anterior to the tendon of piriformis and the sciatic nerve (Fig. 3). The portal was located at a mean distance of $19 \mathrm{~mm}$ (16 to 22) from the ascending branch of the lateral femoral circumflex artery, $40 \mathrm{~mm}(16$ to 70 ) from the sciatic nerve and $36 \mathrm{~mm}$ ( 6 to 85 ) from the superior gluteal artery.

Anterolateral portal (Fig. 4). The trocar passed through the tensor fascia lata and gluteus medius before entering the lateral margin of the capsule. This portal was located at a mean distance of $56 \mathrm{~mm}$ (46 to 78 ) from the lateral femoral cutaneous nerve at its closest point.

Effect of traction $(23 \mathrm{~kg}$ ) on the portals and neurovascular structures. The addition of traction of $23 \mathrm{~kg}$ had little effect on the spatial relationship of the neurovascular structures and their respective portals. With this amount of traction, the mean distance between the anterior portal and the lateral femoral cutaneous nerve was found to be $4.78 \mathrm{~mm}(0$ to 25). This means that the lateral femoral cutaneous nerve moved a mean distance of $0.22 \mathrm{~mm}$ ( 0 to 3 ) closer to the anterior portal. The femoral nerve moved a mean of 6.4 $\mathrm{mm}$ further from the anterior portal. In addition, traction increased the mean distance of the posterolateral portal from the sciatic nerve by $3 \mathrm{~mm}$, from a mean of $40 \mathrm{~mm}$ to $43 \mathrm{~mm}$.

When the traction force was increased by increments of 2 $\mathrm{kg}$ to $64 \mathrm{~kg}$, there was no evidence of damage to the capsule, the labrum or the ligamentum teres as shown by arthrography or arthroscopy. Nor did further anatomical dissection reveal macroscopic evidence of such damage. Histological examination showed no signs of damage to the anterior, posterior or lateral capsule or to the lateral labrum.

\section{Discussion}

In 1931, Burman ${ }^{15}$ wrote of the difficulty in visualising the convex surface of the femoral head arthroscopically, stating that "It is manifestly impossible to insert a needle between the head of the femur and the acetabulum". Because of the bony constraints of the hip, heavy traction has been used to facilitate access. ${ }^{3,5,6,8,16-18}$ The magnitude of the force required to provide sufficient distraction has been reported as being between 300 and $900 \mathrm{~N}$ in an anaesthetised patient. ${ }^{17}$ Byrd et al $^{14}$ described a vacuum phenomenon which occurs after the initial application of traction and further separation of the joint often occurs after cannulation which breaks the seal.

The role of traction in the development of neurovascular injury during arthroscopy of the hip has been discussed by several authors. ${ }^{5,10,11}$ Both Rodeo et $\mathrm{al}^{11}$ and Byrd et $\mathrm{al}^{14}$ found that femoral traction could result in displacement of neurovascular structures. That which was considered to be at most risk of direct injury was the lateral femoral cutaneous nerve which has a few branches located over the sartorial fascia. The application of traction brought the anterior portal closer to the lateral femoral cutaneous nerve by $0.22 \mathrm{~mm}$, which is not significant $(\mathrm{p}>0.05)$.

The other structure at risk from the anterior portal is the femoral nerve. Rodeo et $\mathrm{al}^{11}$ stated that traction may lateralise this nerve and place it in greater danger. However, according to our study, traction produced a significant increase in the safe distance between the portal and the nerve. The nerve lay at a mean distance of $30.4 \mathrm{~mm}$ from the anterior portal, with $23 \mathrm{~kg}$ of traction and $24 \mathrm{~mm}$ without traction. In our study, the distance of the posterior portal from the sciatic nerve increased with traction from a mean of $40 \mathrm{~mm}$ to a mean of $43 \mathrm{~mm}$. Traction may cause neurapraxia because of direct injury to the myelin sheath, but our study did not address this issue, nor were we able to ascertain the contribution of traction to injury to the pudendal nerve against the perineal post, or neurapraxia secondary to ischaemic compression.

The natural history, diagnosis and management of lesions of the acetabular labrum remain obscure. 
Fitzgerald ${ }^{9}$ documented 25 labral tears in 55 patients who had no precipitating trauma to the hip. Hase and Ueo ${ }^{19}$ found labral tears in ten patients only three of whom reported minor trauma; the remaining seven could recall no precipitating event. While the labrum may be vulnerable to relatively minor trauma, as these authors have suggested, our findings did not demonstrate injury from longitudinal distraction of the hip on the fracture table.

No benefits in any form have been received or will be received from a commercial party related directly or indirectly to the subject of this article.

\section{References}

1. Farjo LA, Glick JM, Sampson TG. Hip arthroscopy for acetabular labral tears. Arthroscopy 1999;15:132-7.

2. Bould M, Edwards D, Villar RN. Arthroscopic diagnosis and treatment of septic arthritis of the hip joint. Arthroscopy 1993:9:707-8.

3. Ide T, Akamatsu N, Nakajima I. Arthroscopic surgery of the hip joint. Arthroscopy 1991:7:204-11.

4. Okada Y, Awaya G, Ikeda T, et al. Arthroscopic surgery for synovial chondromatosis of the hip. J Bone Joint Surg [Br] 1989;71-B:198-9.

5. Dorfmann H, Boyer T. Arthroscopy of the hip: 12 years of experience. Arthroscopy 1999;15:67-72.

6. Kuklo TR, Mackenzie WG, Keeler KA. Hip arthroscopy in Legg-Calve-Perthes disease. Arthroscopy 1999;15:88-92.
7. Santori N, Villar RN. Arthroscopic findings in the initial stages of hip osteoarthritis. Orthopedics 1999;22:405-9.

8. McCarthy JC, Mason JB, Wardell SR. Hip arthroscopy for acetabular dysplasia: a pipe dream? Orthopedics 1998;21:977-9

9. Fitzgerald RH Jr. Acetabular labrum tears: diagnosis and treatment. Clin Orthop 1995;311:60-8.

10. Glick JM, Sampson TG, Gordon RB, Behr JT, Schmidt E. Hip arthroscopy by the lateral approach. Arthroscopy 1987;31:4-12.

11. Rodeo SA, Forster RA, Weiland AJ. Neurological complications due to arthroscopy. J Bone Joint Surg [Am]1993;75-A:917-26.

12. Guhl JF, OIdon DW, Sprague NF. Specific complications: elbow, wrist, hip, and ankle. In: Sprague NF III, ed. Complications in arthroscopy. New York: Raven Press, 1989:199-223.

13. Dorfmann H, Boyer T. Hip arthroscopy utilizing the supine position. Arthroscopy 1996;12:264-7.

14. Byrd JWT, Pappas JN, Pedley MJ. Hip arthroscopy: an anatomic study of portal placement and relationship to the extra-articular strucutures. Arthroscopy 1995;11:418-23.

15. Burman MS. Arthroscopy or the direct visualization of joints: an experimental cadaver study. J Bone Joint Surg 1931;29:669-95.

16. Klapper RC, Silver DM. Hip arthroscopy without traction. Contemp Orthop 1989:18:687-93.

17. Blitzer CM. Arthroscopic management of septic arthritis of the hip. Arthroscopy 1993:9:414-16.

18. Eriksson E, Arvidsson I, Arvidsson H. Diagnostic and operative arthroscopy of the hip. Orthop 1986;9:169-76.

19. Hase T, Ueo T. Acetabular labral tear: arthroscopic diagnosis and treatment. Arthroscopy 1999;15:138-41. 